\title{
Bioremediation of Waste in a Porous Medium
}

\author{
Changsheng Chen* \\ Yahoo! Inc., Sunnyvale, CA 94089 \\ and \\ John Chadam ${ }^{\dagger}$ \\ Department of Mathematics \\ University of Pittsburgh, Pittsburgh, PA 15260
}

May 25, 2005

35L60, 35R35, 41A60, 65D30

\begin{abstract}
A simplified model for bacterial remediation of wastes in a porous medium is proposed. The mathematical model consists of a coupled set of nonlinear partial differential equations and an ordinary differential equation. Basic existence, uniqueness and regularity of the system can be proved in three space dimensions, globally in time. The existence of traveling waves can also be established. In a physically relevant limit, a free boundary problem can be formally derived. In this sharp interface limit the shape stability of the moving reaction fronts can be studied using elementary bifurcation analysis. In agreement with industrial observations, these reaction fronts are stable in the early phases of remediation, becoming unstable as the concentration of bacteria becomes high. This destabilizing of the reaction front could lead to undesirable fingering that could result in some of the waste being inaccessible to the bacteria, greatly reducing the effectiveness of this type of remediation process.
\end{abstract}

\section{Introduction}

In this section we describe the bioremdiation process, propose a mathematical model, and outline the main results.

\footnotetext{
*This work summarizes the Ph.D. dissertation research of CC carried out under the supervision of JC who participated in the workshop and presented the results.

†chadam@pitt.edu
} 
Consider a region of soil that has been contaminated by an immobile waste product (e.g., coal tar). In general, soil harbours an enormous number of dormant bacterial species, some of which would eat away the contaminant if additional limiting nutrients (e.g., oxygen), required for this bacteria's growth, were also present. The current industrial strategy then is to flush the contaminated region with nutrients (and perhaps even particular strains of bacteria) so that the activated bacteria will remove the contaminant as part of their growth process, transforming the environmental waste into neutral compounds (e.g., water and carbon dioxide).

Bioremediation was first systematically studied by Borden and Bedient and their co-workers [1, 2]. A discussion of the techological and practical aspects of bioremediation can be found in [3]. The process has been modeled numerically in $[4-7]$ among other workers. One of the first mathematical models for bioremediation was proposed by Odencrantz, Valocchi and Rittmann [8] and was elaborated upon and studied analytically by Oya and Valocchi [9] and by J. Xin and his co-workers $[10-12]$. These works model the biodegredation reaction using Monod kinetics

$$
R=\frac{q c n m}{\left(K_{m}+m\right)\left(K_{n}+n\right)}
$$

where $q$ is the reaction rate coefficient, $c$ is the concentration of bacteria, $n$ is the concentration of nutrient, $m$ is the concentration of the waste, and $K_{m}, K_{n}$ are constants representing the half-maximum rate concentrations of $m$ and $n$ respectively. This model has the property (as observed in nature) that the concentration of the bacteria has an upper bound (see [10]). Moreover, by making a quasi-equilibrium assumption one can reduce the model to a two-component reaction-transport problem in which the concentration of the nutrient and bacteria move along together $[10,11]$.

Practitioners have noticed that the remediation process is effective if the concentrations of injected mobile species do not exceed critical values. At these higher levels they notice that unaltered regions begin to appear. In this note we will propose a new model with a nonlinear reaction-transport feedback mechanism that provides a possible explanation for this undesirable appearance of unaltered zones of contaminated soil.

Suppose that we model the soil as a homogeneous porous medium (i.e., the unaltered zones of interest will not arise as the result of heterogeneities but can only occur spontaneously due to self-organization caused by a destabilizing feedback mechanism). Let $\varphi$ be the porosity of the porous medium, $m(x, t)$ the concentration of the solid, immobile waste material and $c(x, t)$ the aqueous concentration of injected material (nutrients, waste-specific bacteria). Here $t \in[0, \infty)$ is time and $x \in \Omega \subset \mathbf{R}^{3}$ where $\Omega$ is the spatial region of interest. 
The conservation of aqueous species is given by

$$
\frac{\partial(\varphi c)}{\partial t}=\nabla \cdot(\varphi D \nabla c-\varphi c \mathbf{v})-\rho \frac{\partial m}{\partial t}
$$

where $\rho$ is the (constant) density of the solid contaminant, $D$ is the diffusion coefficient and $\mathbf{v}$ the fluid velocity given by Darcy's Law in terms of the pressure $p$

$$
\mathbf{v}=-\frac{\kappa}{\mu} \nabla p
$$

The appearance of the porosity $\varphi$ in equation (1.2) reflects the fact that the fluid can only flow through the pores. In equation (1.3) $\kappa$ is the permeability and $\mu$ is the viscosity. These equations are augmented by the conservation equation for the fluid

$$
\frac{\partial\left(\rho_{f} \varphi\right)}{\partial t}=\nabla \cdot\left(\rho_{f} \varphi \mathbf{v}\right)
$$

where $\rho_{f}$ is the fluid density, and by an equation describing the nutrient/bacteria reaction with the waste;

$$
\frac{\partial m}{\partial t}=-\ell m c\left(c_{e q}-c\right)_{+}
$$

This last equation models one of the simplest types of reaction that captures the dependence on the presence of the contaminant, the presence of the nutrient/bacteria along with a cutoff if the concentration of the nutrient/bacteria exceeds some equilibrium value $c_{e q}$ (as in the preceding models [9 - 11]).

The destabilizing mechanism of interest here does not depend on the specific form of this reaction (see Section 4) so it suffices to present the discussion in terms of the simplest case (1.5). The destabilizing mechanism does, however, depend on the properties of the transport coefficients $\kappa, \mu$ and $D$. To begin, it is not unreasonable for them to be functions of the form $\kappa(c, \varphi), D(c, \varphi)$ and $\mu(c)$. While specific forms for these functions can be found in [13] only general properties will be required for the present discussion. Indeed, we can simplify further by taking the porosity $\varphi$ to be a constant; i.e., the removal of the contaminant does not appreciably change the porosity of the soil (the case of porosity variations caused by changes in $m$ was treated in previous work of one of the authors and his collaborators [14 - 17]). For the present discussion it suffices to consider the two cases of

$$
k(c)=\kappa(c) / \mu(c)
$$

either increasing as function of $c$ or decreasing as a function of $c$. The former case might describe the situation when the concentration $c$ is small allowing the bacteria to swim to areas of more food (waste) in addition to just being 
carried along by the flow. Another low concentration mechanism that might give rise to increasing $k$ is that a developing thin coating of bacteria on an otherwise rough porous matrix would increasingly facilitate the flow of the nutrient/bacteria through the pore channels. At the other extreme, when the concentration $c$ becomes large one can imagine the pores becoming blocked (i.e., $\kappa(c)$ decreasing) and/or the fluid becoming more viscous (i.e., $\mu(c)$ increasing), both leading to $k$ being an decreasing function in this range of concentrations. One can likewise consider a variable diffusion coefficient, $D(c)$ (please see the remarks at the end of Section 4).

Returning to the problem (1.2) - (1.5) with $\varphi$ and $\rho_{f}$ constant, inserting (1.6), and transforming to the scaled variables $\tilde{c}=c / c_{e q}, \tilde{t}=\epsilon t$ with $\epsilon=$ $\varphi c_{e q} / \rho<<1$, one obtains (dropping the tildes)

$$
\begin{array}{r}
\epsilon c_{t}=\nabla \cdot(D(c) \nabla c+c k(c) \nabla \rho)-m_{t} \\
\nabla \cdot(k(c) \nabla \rho)=0, \\
\epsilon m_{t}=-\ell m c(1-c)_{+} .
\end{array}
$$

For specificity we now take the spatial region to be the doubly infinite rectangular cylinder $\Omega=\mathbf{R} \times(0,1)^{2}$. We now impose the following boundary conditions at the inlet $(x=-\infty)$ and the outlet $(x=+\infty)$ of the channel: for $(y, z) \in(0,1)^{2}$ and all $t>0$, at $x=-\infty$

$$
\begin{gathered}
c=c_{i}<1, \\
m=0, \\
\mathbf{v}=-k\left(c_{i}\right) p_{x}=A(t),
\end{gathered}
$$

and at $x=+\infty$

$$
\begin{gathered}
c=1, \\
m=M(\text { constant }), \\
\mathbf{v}=-k(1) p_{x}=A(t) .
\end{gathered}
$$

The first equation in (1.10) says there is a constant inlet concentration that is below the (scaled) equilibrium value. The second says that the remediation has progressed long enough for all of the contaminant to have been removed at the inlet and the reaction zone has now progressed to some point upstream. The third prescribes the inlet fluid velocity via Darcy's Law. Equation (1.10d) says that at the outlet the concentration has reached its equilibrium value after having passed through the entire reaction zone and (1.10e) that the contaminant is still in its original, unaltered state because the reaction zone has not yet reached this point. The last says that the outlet velocity must be the same 
as the inlet velocity because on the boundary of the constant cross-section of the channel $(0,1)^{2}$ we are assuming the no flow conditions

$$
\frac{\partial c}{\partial n}=\frac{\partial p}{\partial n}=0, \quad(y, z) \in(0,1)^{2},
$$

where $n$ is the normal. Finally, we complete the problem by imposing the initial conditions

$$
\begin{gathered}
c_{i} \leq c(x, y, z, 0)=c_{0}(x, y, z) \leq 1, \\
0 \leq m(x, y, z, 0)=m_{0}(x, y, z) \leq M .
\end{gathered}
$$

The model equations (1.7 - 1.11) differ from those in [9 - 12] in several aspects. As mentioned earlier, our reaction (1.9) is simpler than the Monod kinetics but still captures its main features along with the physically important fact that the biomass (bacteria) has an upper bound for its concentration. The most significant difference is that in our model the diffusion and advection coefficients, $D(c)$ and $k(c)$, are concentration dependent. Although this leads to a nonlinear problem, we believe it is essential for the reaction-transport feedback mechanism that causes reaction fronts to destabilize and lead to unaltered contaminant zones as explained below. A second important difference is that we are interested in the shape stability analysis of planar fronts while the previous work examines one-dimensional fronts (planar [9 - 12] and spherical $[18,19])$ and their stability within their own class. As a result our analysis must necessarily be higher dimensional while the previous work is exclusively one-dimensional. In order to explicitly derive traveling wave solutions for the $\epsilon \neq 0$ version of the problem in $[9-12]$ the authors restrict their attention to the purely advective case (i.e., $D \equiv 0$ ), citing the common folklore that diffusion/dispersion has a stabilizing effect, including spreading of the reaction zones. In our work we retain the diffusive/dispersive effects (i.e., $D(c) \neq 0$ ) in order to examine more precisely the resulting stabilizing effects. In [9-12] an explicit traveling wave solution is available but for our more complicated model $(1.7-1.11)$ it is not explicitly available when $D(c) \neq 0$ and $\epsilon \neq 0$. The planar traveling wave solution to problem $(1.7-1.11)$ with $D(c) \neq 0$ can only be calculated explicitly in the sharp interface limit, $\epsilon \rightarrow 0$. As a consequence, we will study its shape stability within the class of sharp interface solutions, i.e., in the context of the moving free boundary problem obtained as the singular limit of problem (1.7 - 1.11) when $\epsilon \rightarrow 0$.

In the next section we summarize our rigorous results concerning the existence, uniqueness, regularity and asymptotic behaviour (as $\epsilon \rightarrow 0$ ) of the solutions to the model equations (1.7 - 11). A formal outline of how to obtain a sharp interface limit $(\epsilon \rightarrow 0)$ to these equations is presented in Section 3. The resulting free boundary problem for the moving reaction interface is amenable to a shape stability analysis based on elementary bifurcation theory. In Section 4 we summarize the conclusions of this stability analysis showing that it 
confirms the previously cited observations of the practitioners. Specifically, we show that if $D$ and $k$ are constant (i.e., there is no feedback between reaction and flow through the concentration dependence of the transport coefficients) then planar reaction fronts remain stable. As a result, the contaminant is removed uniformily over the cross-section by the traveling, planar reaction front. In the "early" phase of the remediation, when the aqueous concentration, $c$, in the reaction zone is low and $k(c)$ is increasing, the reaction front is still stable. When the concentration $c$ is large and pores are increasingly being plugged and the fluid viscosity is rising (i.e., $k(c)$ ) is a decreasing function) the planar reaction fronts lose stability over a bounded interval $(0, A)$ of wavelengths. This simultaneous destabilization of multiple modes can lead to fingering in the reaction front that, in turn, can result in contaminated regions being left unaltered, as observed in practice.

\section{Rigorous Results}

Throughout this discussion we will assume that the transport coefficients are bounded, smooth functions satisfying

$$
0<a \leq D(s), k(s) \leq b \text { for all } s \geq 0
$$

This restricts the porous matrix to never being completely plugged nor completely removed.

Theorem 1 If $c_{0}-c_{i} \in L^{2}($ near $x=-\infty), m_{0} \in L^{1}($ near $x=-\infty), 1-c_{0} \in$ $L^{2}($ near $x=+\infty)$ and $M-m_{0} \in L^{1}($ near $x=+\infty)$ then equations $(1.6-11)$ have a global (in time) weak solution.

The proof involves proving existence for the cutoff problem in $x \in[-N, N]$ by using Schauder's Fixed Point Theorem for our nonlinear parabolic-ellipticordinary system of differential equations. By obtaining appropriate a priori estimates one can take the $N \rightarrow \infty$ limit. The proof models that in $[15$ - 17] (with porosity dependent $D(\varphi), \kappa(\varphi)$ ) but, here, extends to three space dimensions whereas the original, porosity dependent, case has only been solved in two dimensions (i.e., in $(-\infty, \infty) \times(0,1))$. The uniqueness, which is also not available in [15 - 17] follows in this case because of the availability of suitable a priori estimates.

Theorem 2 If $\sup _{\Omega}(|\nabla c|+|\nabla p|) \leq f(t)$ for some increasing continuous function $f$, then the solution is unique.

The proof of the bound in Theorem 2 seems difficult because of the unboundedness of the spatial region. On the other hand, regularity of the solutions is straightforward. 
We now direct our attention to finding traveling wave solutions which are relevant to studying the shape stability of the reaction zone. In particular, we specialize to the one space dimensional (planar) version of the problem and outline the existence of a constant velocity, traveling wave solution for all $\epsilon>0$ using dynamical system methods. In the limit as $\epsilon \rightarrow 0$ we can also obtain an explicit traveling wave solution. This will motivate our study in the next section of the moving free boundary version of the problem.

In terms of the similarity variable $\xi=x-v t$, planar solutions have the form $c(x, y, z, t)=c(\xi)$ (similarly for $m$ and $p$ ). Denoting derivatives with respect to $\xi$ by a prime, equations $(1.7-1.9)$ become

$$
\begin{array}{r}
-\epsilon v c^{\prime}=\left(D(c) c^{\prime}+c k(c) p^{\prime}\right)^{\prime}+v m^{\prime} \\
\left(k(c) p^{\prime}\right)^{\prime}=0, \\
-\epsilon v m^{\prime}=-\ell m c(1-c)_{+} .
\end{array}
$$

These equations are to be solved subject to the boundary (inlet and outlet) conditions

$$
c(-\infty)=c_{i}>0, \quad m(-\infty)=0, \quad c(\infty)=1 \quad m(\infty)=M .
$$

and (e.g., integrating (2.3) from $x=-\infty$ to $x=+\infty$ or using (1.9c) and $(1.9 f))$

$$
v_{i}=-k\left(c_{i}\right) p^{\prime}(-\infty)=-k(1) p^{\prime}(+\infty)=v_{0} .
$$

That is, the inlet velocity $v_{i}$ (equivalently the inlet pressure gradient, $p^{\prime}(-\infty)$ ) are given and the outlet values are determined from (2.6). Integrating (2.2) from $\xi=-\infty$ to $\xi=+\infty$ and using (2.6) one obtains the speed of the travelling wave solution

$$
v=v_{i}\left(1-c_{i}\right)\left(M+\epsilon\left(1-c_{i}\right)\right)^{-1},
$$

or in the original, unscaled variables $t_{\text {orig }}=t / \epsilon$ with $\epsilon=\varphi c_{e q} / \rho$ and $c=$ $c_{\text {orig }} / c_{\text {eq }}$

$$
V=\frac{\varphi}{\rho} v_{i}\left(c_{e q}-c_{i}\right)\left(M+\frac{\varphi}{\rho}\left(c_{e q}-c_{i}\right)\right)^{-1} .
$$

This explicitly shows that in the original coordinates, the velocity of the front is much slower (since $\varphi<<\rho$ ) than the characteristic fluid velocity $v_{i}$. Similarily, integrating from $\xi=-\infty$ to an arbitrary $\xi$ one obtains

$$
c^{\prime}=\frac{1}{D(c)}\left\{\left(v_{i}-\epsilon v\right)\left(c-c_{i}\right)-v m\right\}
$$

which, with equation (2.4), written as

$$
m^{\prime}=\frac{1}{\epsilon v} m c(1-c)_{+},
$$


is the system to be studied. The two relevant fixed points $\left(c_{i}, 0\right)$ and $(1, M)$ are respectively an unstable node and a saddle point. Standard analysis shows that an orbit emanating from the stable manifold of $(1, M)$ tends, as $\xi \rightarrow-\infty$, to $\left(c_{i}, 0\right)$ thus establishing the existence of a solution to equations $(2.2-2.6)$. In summary, one has (up to translations),

Theorem 3 There is a unique traveling wave solution to problem (2.2 - 2.6).

One can also study the $\epsilon \rightarrow 0$ limit for the above solutions $\left(c_{\epsilon}(\xi), m_{\epsilon}(\xi)\right)$ of problem $(2.2-2.6)$. In fact one can obtain the explicit form of this limiting solution.

Theorem 4 Up to translations, $\lim _{\epsilon \rightarrow 0}\left(c_{\epsilon}(\xi), m_{\epsilon}(\xi)\right)$ exists and is equal to $(\bar{c}(\xi), \bar{m}(\xi))$ where

$$
\bar{m}(\xi)=\left\{\begin{array}{lc}
0 & \xi<0 \\
M & \xi>0 .
\end{array}\right.
$$

and

$$
\bar{c}(\xi)=\left\{\begin{array}{l}
c_{i}+\left(1-c_{i}\right) e^{v_{i} \xi / D_{i}} \quad \xi<0, \\
1 \quad \xi>0 .
\end{array}\right.
$$

Here $v_{i}=-\kappa\left(c_{i}\right) p^{\prime}(-\infty)$ and $D_{i}=D\left(c_{i}\right)$ are the inlet velocity and diffusion coefficient.

From an industrial point of view, this is the ideal solution - a planar reaction front moving along with a velocity which is much slower than the fluid velocity, eating away all the contaminant as it advances.

\section{Free Boundary Problem}

In the previous section we outlined the existence of solutions to problem (1.7 1.11) when $\epsilon \neq 0$, including a planar traveling wave solution. We also obtained an explicit sharp interface planar traveling wave solution $(2.9-2.10)$ when $\epsilon \rightarrow 0$. In this section we begin the study of the shape stability of this solution within the class of sharp interface solutions. Specifically, we shall first obtain the sharp interface $(\epsilon \rightarrow 0)$ version of equations $(1.7-1.11)$ as a moving free boundary problem. It will be obvious that $(\bar{c}(\xi), \bar{m}(\xi))$ given by $(2.9,2.10)$ is a solution of these equations. In the following Section 4 we shall outline the shape stability analysis that confirms our observations presented at the end of the first section.

Formally, letting $\epsilon \rightarrow 0$ in equation (1.9), the reaction term on the right hand side vanishes when either $m=0$ or $c=1$ since $c \geq c_{i}>0$. We will denote the interface between these two regions by $x=R(y, z, t)$ - the unknown moving 
free boundary. In the discussion to follow we will restrict our presentation to the two-dimensional case (i.e., all functions are $z$-independent). The same arguments and conclusions apply to the three-dimensional case solely at the expense of more complicated notation.

Following standard asymptotic procedures, we expand all functions $(c, m, p)$ in these two outer regions separated by the reaction interface as follows:

$$
f^{\text {outer }}=f_{0}(x, y, t)+\epsilon^{1 / 2} f_{1}(x, y, t)+o\left(\epsilon^{1 / 2}\right) .
$$

The outer solution (dropping the subscript zero on the leading order term) satisfies the equations:

$$
\left.\begin{array}{l}
\nabla \cdot(D(c) \nabla c+c k(c) \nabla p)=0 \\
\nabla \cdot(k(c) \nabla p)=0 \\
m \equiv 0
\end{array}\right\} \text { in } x<R(y, t)
$$

and

$$
\left.\begin{array}{l}
c \equiv 1 \\
\Delta p=0 \\
m \equiv M
\end{array}\right\} \text { in } x>R(y, t)
$$

Matching these outer solutions across the interface is accomplished by "magnifying" the thin reaction zone, using inner functions to study the relevant internal structure and matching the inner solutions with the outer solutions. Specifically, letting $\sigma$ denote the upstream normal coordinate to the (unknown) interface $x=R(y, t)$, and $\tau$ the tangent coordinate, then with $\eta=\sigma / \sqrt{\epsilon}$ denoting the dilated coordinate, the inner functions have the form

$$
f^{\text {inner }}=\tilde{f}_{0}(\eta, \tau, t)+\epsilon^{1 / 2} \tilde{f}_{1}(\eta, \tau, t)+o\left(\epsilon^{1 / 2}\right)
$$

Substituting these into equations $(1.7-1.10)$, solving for the top order terms and imposing the usual matching conditions

$$
\lim _{\eta \rightarrow \pm \infty} \tilde{f}_{0}(\eta, \tau, t)=\lim _{x \rightarrow R(y, t) \pm 0} f_{0}(x, y, t)
$$

provides the following "jump" conditions at the interface $x=R(y, t)$

$$
\begin{gathered}
c_{-}=c_{+}(\equiv 1) \\
p_{-}=p_{+} \\
\left(\frac{\partial p}{\partial n}\right)_{-}=\left(\frac{\partial p}{\partial n}\right)_{+} \\
D(c)\left(\frac{\partial c}{\partial n}\right)_{-}=M V_{n}
\end{gathered}
$$


where subscripts - and + indicate values downstream and upstream of the front, $n$ is the normal in the direction of $\sigma$ and $V_{n}$ is the normal velocity of the front. Evidently, only the concentration gradient and the waste concentration

are discontinuous across the front $\left(\right.$ since $\left(\frac{\partial c}{\partial n}\right)_{+}=0$ because $c_{+} \equiv 1$ and $m_{-}=0, m_{+}=M$.) The motion of the interface is driven through condition (3.8d) containing these discontinuities. Equations (3.2 - 3.8) provide the sharp interface (moving free boundary) version of problem $(1.7-1.11)$.

Recall that in Section 2 we outlined the proof of the existence of planar traveling wave solutions to equations $(1.7-1.11)$ for all $\epsilon>0$. In addition, we pointed that when $\epsilon \rightarrow 0$ in this class of planar solutions we obtained the solution $(2.9,2.10)$

$$
\begin{gathered}
\bar{m}(x, t)= \begin{cases}0 & x<v t \\
M & x>v t .\end{cases} \\
\bar{c}(x, t)=\left\{\begin{array}{cc}
c_{i}+\left(1-c_{i}\right) \exp \left(v_{i} / D_{i}\right)(x-v t) \quad x<v t \\
1 & x>v t .
\end{array}\right. \\
\bar{p}(x, t)= \begin{cases}p_{0}-\int_{0}^{x-v t} \frac{v_{i}}{k(\bar{c}(s))} d s & x<v t \\
p_{0}-v_{i}(x-v t)_{k_{i}} & x>v t .\end{cases}
\end{gathered}
$$

where $p_{0}$ is an arbitrary constant (pressure at the interface) and $v$ is the unique travelling wave velocity obtained in (2.6a) with $\epsilon=0$;

$$
v=v_{i}\left(1-c_{i}\right) / M
$$

The form of the pressure solution is obtained by integrating (2.3) with (3.9b) substituted. It is a straightforward calculation to check that (3.9) is a planar solution to the free boundary problem $(3.2-3.8)$. Indeed it can be shown to be the unique (up to translations) planar solution. In the next section, we will investigate its stability within the class of all sharp interface $(\epsilon=0)$ solutions; i.e., within the class of solutions to the free boundary problem (3.2 - 3.8).

\section{Shape Stability Analysis}

For notational convenience we let the size of the transverse dimension of the aquifer be $\pi$ and translate the moving front to the origin by the change of variables $x^{\prime}=x-v t$ (and drop the prime). One can then study the stability of small perturbations of the planar front (3.9) of the form

$$
\begin{aligned}
& c(x)=\bar{c}(x)+\delta e^{\sigma t} c_{1}(x) \cos \ell y \\
& p(x)=\bar{p}(x)+\delta e^{\sigma t} p_{1}(x) \cos \ell y
\end{aligned}
$$




$$
R(y, t)=0+\delta e^{\sigma t} \cos \ell y
$$

where $\delta$ is a small parameter, $\ell=1,2, \cdots$ is the wave number of the shape disturbance and $\sigma=\sigma(\ell)$ is the spectrum of the linearized problem. With the notation $D=D(\bar{c}), k=k(\bar{c}), D^{\prime}=D^{\prime}(\bar{c})$, etc., the linearized problem for $c_{1}, p_{1}$ can be written as: in $x<0$

$$
\begin{array}{r}
D c_{1}^{\prime \prime}+\left(2 D^{\prime} \bar{c}^{\prime}+k \bar{p}^{\prime}\right) c_{1}^{\prime}+\left(D^{\prime \prime}\left(\bar{c}^{\prime}\right)^{2}-\ell^{2} D+D^{\prime} \bar{c}^{\prime \prime}\right) c_{1}+k^{\prime} \bar{c}^{\prime} \bar{p}^{\prime} c_{1}+\ell^{2} D \bar{c}^{\prime}+k \bar{c}^{\prime} \bar{p}_{1}^{\prime}=0 \\
k\left(p_{1}^{\prime \prime}-\ell^{2} p_{1}+\ell^{2} \bar{p}^{\prime}\right)+\left(k \bar{p}^{\prime \prime}+k^{\prime \prime} \bar{c}^{\prime} \bar{p}^{\prime}\right) c_{1}+k^{\prime} \bar{p}^{\prime} c_{1}^{\prime}+k^{\prime} \bar{c}^{\prime} p_{1}^{\prime}=0
\end{array}
$$

and in $x>0$

$$
\begin{gathered}
c_{1} \equiv 0 \\
p_{1}^{\prime \prime}-\ell^{2} p_{1}+\ell^{2} \bar{p}^{\prime}=0,
\end{gathered}
$$

along with the asymptotic conditions

$$
\begin{gathered}
c_{1} \rightarrow 0, \quad p_{1}^{\prime} \rightarrow 0 \quad \text { as } x \rightarrow-\infty \\
p_{1}^{\prime} \rightarrow 0 \quad \text { as } x \rightarrow+\infty
\end{gathered}
$$

and the conditions on the interface $x=0$

$$
\begin{gathered}
c_{1-}=0 \\
p_{1-}=p_{1+} \\
p_{1-}^{\prime}=p_{1+}^{\prime} \\
D(1) c_{1-}^{\prime}=\sigma M .
\end{gathered}
$$

Equations (4.2 - 4.5) cannot be solved explicitly for arbitrary $D(c)$ and $k(c)$ and hence an expression for the spectrum is not available in general. Instead we summarize the stability results for a few illustrative cases that support our observations and objectives mentioned in the first section.

Example 1. $D(c) \equiv 1 \equiv k(c)$. This is the prototype of the case of constant (concentration independent) transport coefficients whose importance for this discussion is that all interaction between the reaction and the transport (through the concentration $c$ ) is precluded. Direct calculation shows that

$$
p_{1}^{\prime}(x)=0, \quad-\infty<x<\infty
$$

and in $x<0$

$$
c_{1}(x)=v_{i}\left(1-c_{i}\right) e^{v_{i} x}\left(1-\exp \left(\frac{\sqrt{v_{i}^{2}+4 \ell^{2}}-v_{i}}{2} x\right) .\right.
$$


Substituting (4.6b) into the interface condition (4.5d) gives for the spectrum of the $\ell^{\text {th }}$ mode

$$
\sigma(\ell)=\frac{v_{i}\left(1-c_{i}\right)}{M}\left(v_{i}-\left(v_{i}+\sqrt{v_{i}^{2}+4 \ell^{2}}\right) / 2\right)
$$

which is clearly negative for each mode $\ell=1,2, \cdots$ and hence the planar front (3.9) is stable. Thus when there is no possibility of feedback between the reaction and the transport, the planar remediation front is stable to heterogeneous perturbations and moves through the aquifer, uniformly removing the contaminant. This is reminiscent of the (stable) Stefan problem modeling the melting of ice.

Example 2. $D(c) \equiv 1, k(c)=e^{a c}$ with $a>0$. This corresponds to "early stage" remediation when $k(c)$ is increasing with $c$ and, on phenomenological grounds, we expect the remediation front to remain stable. Following [18], we write equations (4.2) and (4.3) in terms of the horizontal velocity in $x<0$

$$
u=-k(c) p_{x}=-k(\bar{c}) \bar{p}^{\prime}+\delta u_{1} e^{\sigma t} \cos \ell y
$$

and eliminating $c_{1}$, one obtains for $u_{1}$ the problem

$$
\left(\frac{d^{2}}{d x^{2}}-\ell^{2}-v_{i} \frac{d}{d x}\right)\left(\frac{d^{2}}{d x^{2}}-\ell^{2}\right) u_{1}-a\left(1-c_{i}\right) v_{i} e^{v_{i} x}\left(\frac{d}{d x}+v_{i}\right)\left(\frac{d^{2}}{d x^{2}}-\ell^{2}\right) u_{1}=0
$$

along with the boundary conditions as $x \rightarrow-\infty$

$$
u_{1} \rightarrow 0, \quad u_{1}^{\prime \prime} \rightarrow 0
$$

and on the interface $x=0$

$$
u_{1}^{\prime}=-\ell u_{1}, u_{1}^{\prime \prime}=a \ell\left(1-c_{i}\right) v_{i}\left(u_{1}-\ell v_{i}\right)+\ell^{2} u_{1} .
$$

Thus the simple change of allowing $k$ to be $c$-dependent has led to a complicated boundary value problem $(4.9,10)$ for the stability analysis. The equations become a bit more transparent by making the change of variables

$$
t=-a\left(1-c_{i}\right) e^{v_{i} x}, \quad y=\left(\frac{d^{2}}{d x^{2}}-\ell^{2}\right) u_{1}
$$

that results in $y$ satisfying the Kummer equation

$$
\frac{d^{2} y}{d t^{2}}+\frac{d y}{d t}+\left(\frac{1}{t}-\left(\frac{\ell}{v_{i}}\right)^{2} / t^{2}\right) y=0 .
$$

One finds that the solution can be written as

$$
y(t)=(-t)^{\mu+1 / 2} e^{-t} M(\mu-1 / 2,1+2 \mu, t)
$$

where $\mu=\left(\left(\ell / v_{i}\right)^{2}+1 / 4\right)^{1 / 2}$ and $M$ is a Kummer function. Solving for $u_{1}$ (in 4.11 ), for $c_{1}$ (from (4.2a)) and substituting into the boundary condition (4.5d) one obtains 


$$
\begin{aligned}
\sigma(\ell)= & \frac{\left(1-c_{i}\right)}{M \ell^{2}}\left\{\ell^{2} v_{i}^{2}+a_{2} \frac{d y}{d t}\left(-a\left(1-c_{i}\right)\right)\right. \\
& \left.+a_{2} y\left(-a\left(1-c_{i}\right)\right)+a_{2} a_{1}\left(1-v_{i}\right)\left[2 v_{i}\left(a\left(1-c_{i}\right)\right)^{\ell / v_{i}}\right]\right\}
\end{aligned}
$$

where the constants $a_{1}, a_{2}$ are given by

$$
\begin{gathered}
a_{1}=\int_{-a\left(1-c_{i}\right)}^{0}(-s)^{\ell / v_{i}} s^{-1} y(s) d s \\
a_{2}=\frac{2 a\left(1-c_{i}\right) \ell^{2} v_{i}^{2}}{a_{1}\left[a\left(1-c_{i}\right)\right]^{-\left(\ell / v_{i}+1\right)}+y\left(-a\left(1-c_{i}\right)\right)} .
\end{gathered}
$$

Using properties of Kummer functions [19] one can numerically evaluate $\sigma(\ell)$ in (4.14) and find that for every $\ell=1,2, \cdots, \sigma(\ell)$ is negative. This again implies that the planar front is stable.

Example 3. $D \equiv 1, k(c)=e^{a c}, a<0$. This corresponds to "late stage" remediation when blockage begins to occur and we expect the remediation front to be unstable. Clearly, the analysis outlined in Example 2 obtains with $a<0$. The expression (4.14) now gives an interval $(0, A)$ of values of the wave number $\ell$ for which $\sigma(\ell)>0(\sigma(0)=\sigma(A)=0)$. The size of the unstable interval, $A$, depends in a monotonically increasing manner on the Peclet number, $v_{i} L / D_{i}$, where $L$ is the traverse width of the channel and $D_{i}$ is the inlet diffusion coefficient. Indeed, the Peclet number is the bifurcation parameter. Thus, depending on the size of the Peclet number, one might have a large number of unstable models that could combine to lead to fingering in the front. This in turn may lead to the phenomenon observed by practitioners that some regions are left unaltered by the remediation process.

Example 4. $D(c)=1+h c, k=e^{a c}$. This example is intended to examine the effect of a variable diffusion coefficient on the above stability results. Unfortunately, closed solutions are not available in this case nor for $D(c)=\exp (h c)$. All of the results are obtained from numerical simulations based on shooting methods for solving the boundary value problems. As a test we checked that the algorithms confirm the results of the previous three examples when $h=0$. We find that $h>0$ (i.e., $D(c)$ increases with $c$ ) enhances stability and $h<0$ (i.e., $D(c)$ decreases with $c$ ) enhances instability. This agrees with the qualitative statements in $[9-12]$ and with the earlier comment that the Peclet number is the bifurcation parameter (note that the diffusion appears in the denominator). On the other hand, for $|h|<1$ our numerical experiments never show that a qualitative change from the $D \equiv 1$ case can be effected by a change in $h$. That is, the situation in Example 2 of stability for $D \equiv 1, k(c)=e^{a c}, a>0$, 
cannot lead to instability by taking $D(c)=1+h c, h<0$. More specifically, in this case $\sigma(\ell)$ rises but remains negative for all $\ell$. Similarly, in Example 3 the interval of instability for $D \equiv 1, k(c)=e^{a c}, a<0$, shrinks but does not disappear by taking $D(c)=1+h c, h>0$.

Finally, we mention that there is a wealth of information on experiments, modeling, numerical simulation and analytical treatments of various aspects of remediation in the proceedings of a recent IMA workshop on the subject $[20]$.

\section{References}

[1] R.C. Borden and P.B. Bedient, Transport of dissolved hydrocarbons influenced by oxygen-limited biodegradation, 1, Theoretical development Water Res. Research, 22 (1986) pp. 1973-1982.

[2] R.C. Borden, P.B. Bedient, M.D. Lee, C.H. Ward and J.T. Wilson, Transport of dissolved hydrocarbons influenced by oxygen-limited biodegradation, 2, Field application, Water Res. Research, 22 (1986), pp. 1983-1990.

[3] National Research Council, In Situ Remediation, When Does It Work?, Water Science and Technology Board, National Academy Press, Washington, D.C., 1993.

[4] F.J. Molz, M.A. Widdowson and L.D. Benefield, Simulation of microbial growth dynamics, coupled to nutrient and oxygen transport in porous media, Water Res. Research, 22 (1986), pp. 1207-1216.

[5] K. MacQuarrie and E. Sudicky, Simulation of biodegradable organic contaminants in groundwater, Water Res. Research, 22 (1990), pp. 1207-1216.

[6] C. Chiang, C. Dawson and M. Wheeler, Modeling of in-situ bioremediation of organic compounds in groundwater, Transport in Porous Media, 6 (1991), pp. 667-702.

[7] T.R. Ginn, C.S. Simmons and B.D. Wood, Stochastic-convective transport with nonlinear reaction: Biodegradation with microbial growth, Water Res. Research, 31 (1995), pp. 2689-2700.

[8] J. Odencrantz, A. Valocchi and B. Rittmann, Modeling the interaction of sorption and biodegradation on transport in groundwater in situ bioremediation systems, in Proceedings of the 1993 Groundwater Modeling Conference, E. Poeter et al., eds., Golden, CO, 1993. 
[9] S. Oya and A. Valocchi, Characterization of traveling waves and analytical estimation of pollutant removal in one-dimensional subsurface bioremediation modeling, Water Res. Research, 33 (1997), pp. 1117-1127.

[10] J. Xin and D. Zhang, Statistical analysis of biodegradation fronts in onedimensional heterogeneous porous media, Advances in Water Resources, 22 (1998), pp. 103-116.

[11] R. Murray and J. Xin, Existence of traveling waves in a biodegradation model for organic contaminants, SIAM J. Math. Anal., 30 (1999), pp. $72-94$.

[12] J.X. Xin and J.M. Hyman, Stability, Relaxation, and Oscillation of Biodegradation Fronts, SIAM J. Applied Math., 61, No. 2, (2000), pp. 472-505.

[13] J. Bear, Dynamics of Fluids in Porous Media, American Elsevier, Amsterdam, 1972.

[14] J. Chadam, P. Ortoleva and A. Sen, Reactive percolation instabilities, IMA J. Appl. Math., 36 (1987), pp. 207-220.

[15] J. Chadam, X. Chen, E. Comparini and R. Ricci, Traveling wave solutions of a reaction-infiltration problem and a related free boundary problem, Euro. J. Appl. Math., 5 (1994), pp. 255-265.

[16] X. Chen and J. Chadam, A reaction-infiltration problem I: Existence, uniqueness, regularity and singular limit in one space dimension, Nonlin. Anal. Theory, Meths. and Applics., 27 (1996), pp. 463-491.

[17] X. Chen, R. Gianni, R. Ricci and J. Chadam, A reaction-infiltration problem: Existence, uniqueness and regularity of solutions in two space dimensions, Math. Models and Meths. in Appl. Sci., 5 (1995), pp. 599-618.

[18] S. Oya and A. Valocchi, Analytical approximation of biodegradation rate for an in situ bioremediation of groundwater under ideal radial flow conditions, J. Contam. Hydrology, 31 (1998), pp. 275-293.

[19] J. Xin, Fluid Limit and Free Boundaries in Biodegradation, in Free Boundary Problems: Theory and Applications, N. Kenmochi, ed., GAKUTO Internat. Ser. Math. Sci. Appl., 13 Gakkōtosho, Tokyo, pp. 429-440.

[20] J. Chadam, A. Peirce and P. Ortoleva, Stability of reactive flows in porous media: Coupled porosity and viscosity changes, SIAM J. Appl. Math., 51 (1991), pp. 684-692. 
[21] M. Abramowitz and I.A. Stegun, Handbook of Mathematical Functions, U.S. Government Printing Office, Washington, D.C., 1964.

[22] Resource Recovery, Confinement, and Remediation of Environmental Hazards, J. Chadam, A. Cunningham, R. Ewing, P. Ortoleva and M. Wheeler eds., Volume 131, IMA Volumes in Mathematics and its Applications, Springer-Verlag, New York, Berlin, Heidelberg, 2000. 